\title{
Associations of clinical and imaging characteristics with collateral grade on baseline CT angiography in patients with acute ischemic stroke due to large vessel occlusion
}

Eveline Wiegers ${ }^{1}$, Bob Roozenbeek ${ }^{2,4}$, Maxim Mulder ${ }^{2,4}$, Ivo Jansen ${ }^{3}$, Esmee Venema ${ }^{1,2}$, Kars Compagne ${ }^{4}$, Olvert Berkhemer2,4,5, Bart Emmer ${ }^{4}$, Henk Marquering ${ }^{3}$, Adriaan van $\mathrm{Es}^{4}$, Marieke Sprengers ${ }^{3}$, Yvo Roos ${ }^{6}$, Charles Majoie ${ }^{3}$, Hester Lingsma ${ }^{1}$, Diederik Dippel ${ }^{2}$, Aad van der Lugt ${ }^{4}$

${ }^{1}$ Department of Public Health, Erasmus MC University Medical Center, Rotterdam, the Netherlands; ${ }^{2}$ Department of Neurology, Erasmus MC University Medical Center, Rotterdam, the Netherlands; ${ }^{3}$ Department of Radiology, Academic Medical Center, Amsterdam, the Netherlands; ${ }^{4}$ Department of Radiology and Nuclear Medicine, Erasmus MC University Medical Center, Rotterdam, the Netherlands; ${ }^{5}$ Department of Radiology, Maastricht University Medical Center, Maastricht, the Netherlands; ${ }^{6}$ Department of Neurology, Academic Medical Center, Amsterdam, the Netherlands.

\section{BACKGROUND}

Poor cerebral collateral circulation is associated with poor functional outcome after ischemic stroke and reduces the beneficial effect of endovascular treatment.

\section{AIM}

We aimed to identify clinical and imaging characteristics that are associated with collateral grade on baseline CTA in patients with acute ischemic stroke due to a proximal intracranial occlusion in the anterior circulation.

\section{METHODS}

- Data from the MR CLEAN trial $(n=500)$ and the MR CLEAN Registry ( $n=1488)$, total $n=1988$

- CTA collateral grade was scored on a 4-point scale (Panel)

- Univariable and multivariable ordinal logistic regression analysis to assess the association between CTA collateral grade and characteristic previously suggested in the literature

- Characteristics with $P<0.157$ in univariable analysis were included in the final multivariable regression model

\section{RESULTS}

Table 1. Clinical and imaging characteristics at baseline.

\begin{tabular}{|ll|}
\hline Age - median (IQR) & $69(58-79)$ \\
Male sex - n. (\%) & $1086(55)$ \\
NIHSS - median (IQR) & $16(12-20)$ \\
Systolic blood pressure - mean mmHg (SD) & $149(24)$ \\
Diastolic blood pressure - mean mmHg (SD) & $82(15)$ \\
History of hypertension - n. (\%) & $972(49)$ \\
History of diabetes mellitus - n. (\%) & $323(16)$ \\
History of myocardial infarction - n. (\%) & $302(15)$ \\
History of ischemic stroke - n. (\%) & $303(15)$ \\
Use of statins - n. (\%) & $665(34)$ \\
Use of antiplatelets - n. (\%) & $637(32)$ \\
ICA-(T) occlusion - n. (\%) & $533(28)$ \\
\hline
\end{tabular}

Table 2. Collateral grades on baseline CTA.

\begin{tabular}{|lll|}
\hline Grade 0 & $\mathrm{n}=123$ & $7 \%$ \\
Grade 1 & $\mathrm{n}=596$ & $32 \%$ \\
Grade 2 & $\mathrm{n}=734$ & $39 \%$ \\
Grade 3 & $\mathrm{n}=422$ & $23 \%$ \\
\hline
\end{tabular}

Table 3. Associations of various characteristics with baseline CTA collateral grade. Adjusted common odds ratios $<1$ represent associations with poorer collateral grades.

\begin{tabular}{|c|c|c|}
\hline Characteristic & Adjusted common OR & P-value \\
\hline Age (per 10 years) & $0.88(0.83-0.94)$ & $<0.001$ \\
\hline Male sex & $0.71(0.58-0.80)$ & $<0.001$ \\
\hline History of ischemic stroke & $0.83(0.71-1.15)$ & 0.12 \\
\hline $\begin{array}{l}\text { Baseline diastolic blood pressure } \\
\text { (per } 10 \mathrm{mmHg} \text { ) }\end{array}$ & $0.94(0.90-1.00)$ & 0.03 \\
\hline Use of statins & $0.90(0.74-1.09)$ & 0.28 \\
\hline Use of antiplatelets & $0.85(0.70-1.04)$ & 0.11 \\
\hline ICA-(T) occlusion & $0.58(0.49-0.70)$ & $<0.001$ \\
\hline
\end{tabular}

\section{CONCLUSIONS}

- Older age, male sex, higher baseline diastolic blood pressure, and occlusion of the ICA-(T) are associated with poorer CTA collateral grades.

- No independent association was found with cerebrovascular risk factors, such as hypertension, diabetes, or extracranial carotid stenosis

Panel: Scoring of CTA collateral grade

Grade 0: absent collaterals $0 \%$ filling of the occluded territory

Grade 1: poor collaterals

$>0 \%$ and $\leq 50 \%$ filling of the occluded territory Grade 2: moderate collaterals

$>50 \%$ and $<100 \%$ filling of the occluded territory Grade 3: good collaterals

$100 \%$ filling of the occluded territory

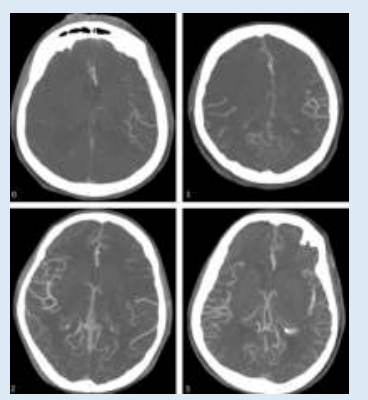

